\title{
9
}

\section{INDICADORES DE LA OCDE SOBRE LA REGULACIÓN DEL MERCADO DE PRODUCTOS (PMR) Resultados de España en 2018}

El conjunto de indicadores Product Market Regulation (PMR) de la OCDE se actualiza cada cinco años y tiene por objeto cuantificar las trabas y barreras generadas tanto por la regulación como por las actuaciones del sector público sobre la economía, haciendo especial énfasis en aspectos procompetitivos. En los resultados de 2018, España se sitúa en segunda posición en el indicador general junto a Dinamarca, por detrás de Reino Unido, con una puntuación de 1,07 (el valor cero corresponde a una regulación menos restrictiva y el seis a la más restrictiva). A pesar de los buenos resultados obtenidos, se detectan sectores en los que siguen existiendo barreras o regulaciones restrictivas.

Palabras clave: competencia, mercados de bienes y servicios, política económica.

Clasificación JEL: H00, K23.

\section{Introducción. La importancia de la buena regulación del mercado de productos y servicios}

Para el buen funcionamiento de los mercados de productos y servicios es esencial la intervención del sector público a través de la regulación. En general, los aspectos institucionales y las políticas económicas generan divergencias en los niveles de productividad entre los países (Conway et al., 2006). Por lo tanto, la influencia de la regulación sobre el

\footnotetext{
* Dirección General de Política Económica. Ministerio de Economía y Empresa.

Este artículo ha sido elaborado por Míriam Segovia Moreno, Jefa de Servicio y Pilar Palacios Guillén, Consejera Técnica.

Versión de septiembre de 2019.

DOI: https:/doi.org/10.32796/bice.2019.3116.6913
}

crecimiento económico da lugar a efectos diferenciadores, tanto positivos como negativos, en las economías.

En concreto, las reformas procompetitivas de política económica y la progresiva liberalización sectorial inciden positivamente sobre la actividad de los agentes en el mercado (Nicoletti y Scarpetta, 2003), alterando el comportamiento de los grupos de interés, las relaciones de agencia o reduciendo la búsqueda de rentas, lo que genera ganancias de eficiencia. Dichas ganancias pueden ser tanto estáticas, relacionadas con la eliminación de la ineficiencia-X (Leibenstein, 1966) o con la reducción de poder de mercado de un monopolista, como dinámicas, dado que las empresas adecuarán su comportamiento por presiones procompetitivas (Winston, 1993; Meyer y Vickers, 1997). $\triangleright$ 
En un contexto dinámico, las ganancias de eficiencia pueden generar incentivos a innovar, favoreciendo la entrada progresiva de empresas e impulsando la productividad.

No obstante, en ocasiones, el impacto de la introducción de reformas con afán liberalizador se puede ver afectado por la existencia de un exceso de cargas regulatorias que ralentice la difusión de shocks positivos de productividad o que genere una ineficiente distribución de recursos. Por ello, siguiendo las tendencias de la better regulation (Informe Mandelkern, 2001) y smart regulation (Gunningham et al., 1998), se hace necesario complementar las reformas con mejoras regulatorias que permitan simplificar la regulación y eliminar el exceso de cargas administrativas (por ejemplo, a través de la sustitución de autorizaciones por declaraciones responsables o comunicaciones, de la elaboración de memorias de evaluación de impacto normativo o del recurso a la regla «one in, one out» ${ }^{1}$, etcétera). Para minimizar estos efectos negativos, desde los años ochenta los policymakers han revisado y actualizado los marcos regulatorios en aras de reducir aquellas normas restrictivas que tendían a imponer excesivas cargas.

Dado que los efectos de la regulación no tienen por qué ser positivos per se, es necesario realizar un análisis de la misma y de su impacto. Para valorar la regulación de los distintos países y poder hacer un seguimiento de las reformas implementadas a lo largo del tiempo, la Organización para la Cooperación y Desarrollo Económico (OCDE) creó en 1998 los indicadores de regulación del mercado de productos (PMR, por sus siglas en inglés), que consisten en un indicador general de la

La regla "one in, one out» implica que, cada vez que se introduzca una nueva carga administrativa se debe proceder a suprimir otra. economía (economy-wide) sobre la intensidad de la regulación del mercado de productos (Nicoletti et al., 2000) que se completa con un conjunto de indicadores que miden la regulación a nivel sectorial (denominados indicadores sectoriales). La evaluación comparativa de diferentes regulaciones y diseños de política económica incentiva a los países a realizar reformas estructurales que favorecen la actividad económica. En junio de 2019 se han publicado los últimos resultados de los PMR referidos a la regulación de los mercados de productos en 2018 (OCDE, 2019).

A lo largo del presente artículo se analizarán los indicadores PMR y se realizará una revisión de la situación de España, con especial atención a sus resultados en 2018 junto con una comparativa sectorial entre 2013 y 2018. Por último, se señalarán aquellos sectores para los que los datos de los PMR sugieren una mayor necesidad de mejorar la regulación.

\section{Descripción de indicadores y metodología}

Los PMR se ocupan de medir hasta qué punto la regulación del mercado de productos y servicios está diseñada para minimizar las distorsiones a la competencia, permitiendo identificar áreas de potencial reforma, así como aportando evidencias de la convergencia en términos regulatorios entre países.

La característica principal de los indicadores PMR de la OCDE es que se centran en la normativa de una manera objetiva, es decir, no se basan en opiniones, sino en normas. Además, se construyen siguiendo un enfoque de «abajo-arriba» en el que se atribuye una puntuación a las distintas medidas que configuran una política. No obstante, estos indicadores $\triangleright$ 
también presentan limitaciones, dado que no pueden cuantificar elementos subjetivos 0 informales como la orientación y asesoría en cuestiones administrativas, así como los efectos del poder sancionador ejercido por las autoridades competentes.

Actualmente, muchas instituciones internacionales como la Comisión Europea o el Fondo Monetario Internacional utilizan estos indicadores para hacer recomendaciones de política económica; por ello, es importante prestar la debida atención a su cumplimentación.

En cuanto a su configuración, como avanzábamos, los PMR están formados por un indicador general y varios sectoriales. El indicador general está compuesto por dos subconjuntos de indicadores o indicadores de nivel alto: por un lado, el indicador «Distorsiones inducidas por el Estado»y, por otro, el indicador «Barreras a la entrada de productos tanto nacionales como extranjeros". A su vez, cada uno de los indicadores de nivel alto se subdivide en tres indicadores de nivel medio y varios indicadores de nivel bajo (Esquema 1).

Por otro lado, hay una clasificación que atiende a criterios sectoriales (Esquema 2), construyéndose indicadores individuales y generales por sector para servicios comerciales e industrias de red ${ }^{2}$. En total hay dieciséis indicadores individuales que reflejan la regulación de diferentes sectores de la economía.

La puntuación obtenida, tanto para los indicadores generales como para los sectoriales, es la media aritmética de los indicadores que los componen y tendrá un valor entre cero y $\triangleright$

2 En los últimos resultados de los PMR no se ha publicado un indicador general para servicios profesionales porque la disparidad de profesiones existentes entre los países analizados no permite realizar comparaciones.

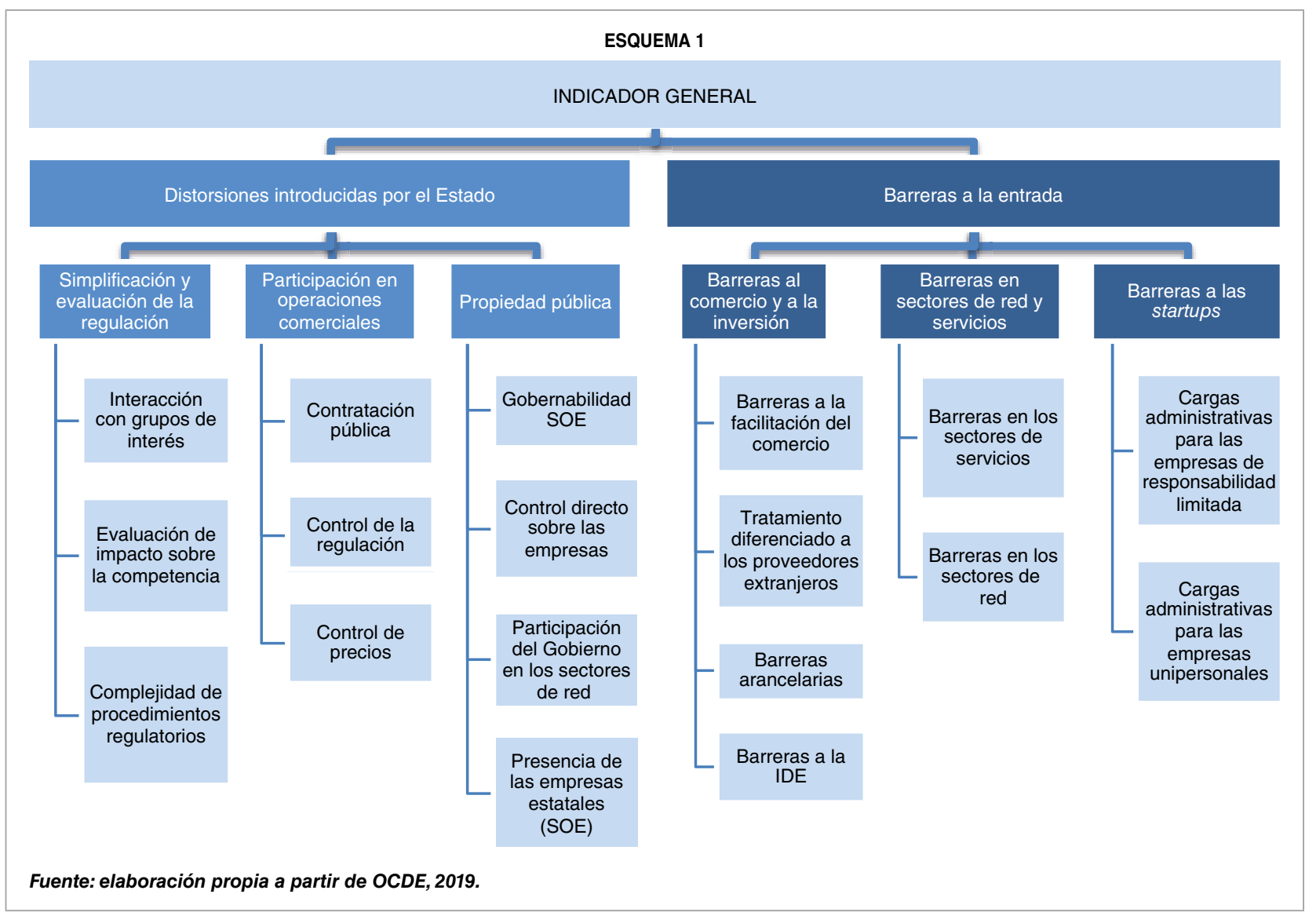




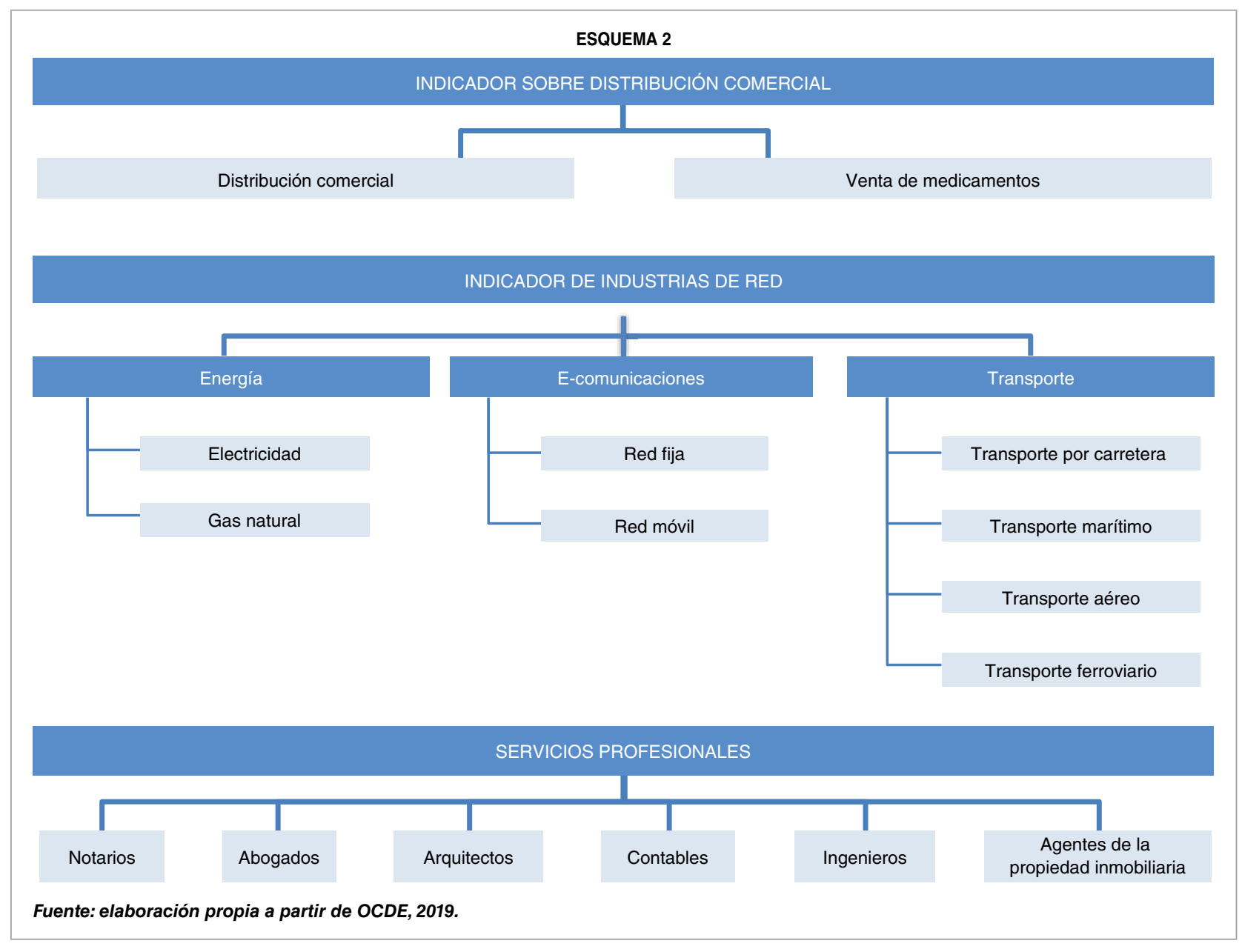

seis en función de lo restrictiva que sea la regulación.

Para la elaboración de estos indicadores la OCDE envía cada cinco años un conjunto de cuestionarios a los países participantes ${ }^{3}$. Estos recogen más de 2.500 preguntas agrupadas en diversas materias ${ }^{4}$. La información aportada por los Gobiernos a través de los cuestionarios está sujeta a un proceso de revisión y contraste por parte de la OCDE. A diferencia de otros

\footnotetext{
3 Generalmente participan los países miembros de la OCDE (salvo alguna excepción) y algún país no perteneciente a la organización. En el año 2018 Estados Unidos y Estonia no completaron los cuestionarios, por lo que no han sido incluidos en los PMR de 2018.

4 Agua, cargas administrativas a la creación de empresas, comercio exterior e inversiones, comercio minorista, comunicaciones electrónicas, contratación pública, diseño regulatorio, economía digital, energía, gobernanza de empresas públicas, otros sectores, profesiones, taxis y transporte.
}

indicadores, los PMR son cumplimentados únicamente por los Gobiernos de cada país, que aportan información sobre el marco regulatorio vigente a fecha 1 de enero del año establecido como referencia.

Es importante señalar que en 2018 se ha realizado una serie de cambios metodológicos que impiden una comparación estricta de los resultados con los de años anteriores. Pasados veinte años desde su diseño inicial, era necesario hacer una profunda revisión para mantener la utilidad de estos indicadores (OCDE, 2018), por lo que se modificó de manera sustancial la estructura del indicador general para adaptarla a la nueva información recogida, asegurando el equilibrio entre los distintos indicadores y manteniendo cierta coherencia $\triangleright$ 


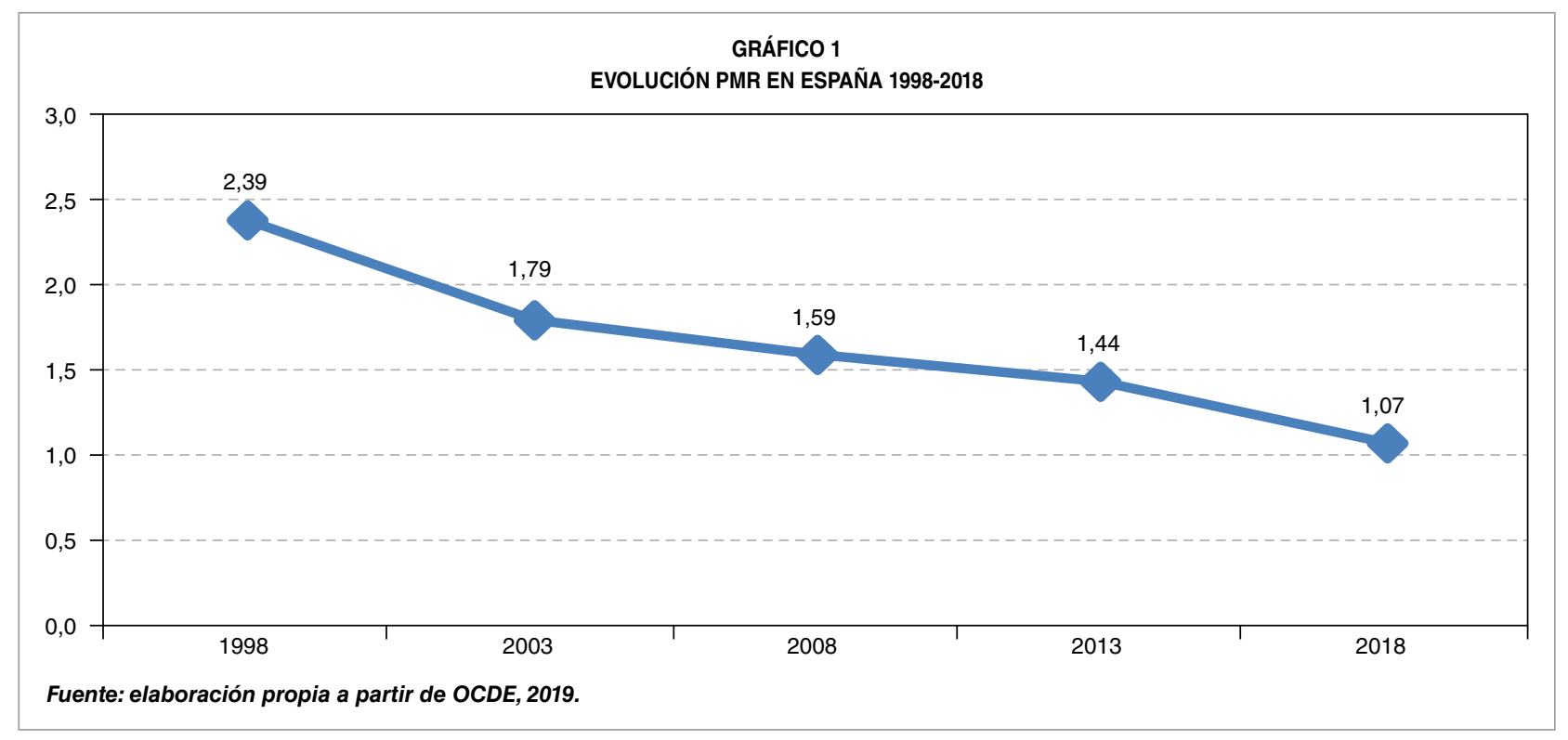

con las versiones anteriores para evitar sesgos en el valor final del indicador.

Entre los cambios introducidos en los cuestionarios destacan:

- La introducción de un área transversal sobre simplificación y evaluación de la regulación.

- La inclusión de notarios y agentes de la propiedad inmobiliaria entre las profesiones a examinar.

- Nuevas preguntas sobre diseño de la regulación de contratación pública y distribución de medicamentos al por menor, así como sobre taxis y otro tipo de arrendamientos de vehículos con conductor para transporte urbano de pasajeros y transporte de viajeros de larga distancia.

\section{Evolución de España de 1998 a 2018. Resultados 2018}

La posición de España ha mejorado considerablemente desde que se pusieron en marcha los PMR. En 1998 España se consideraba un país relativamente restrictivo (Conway, Janod y Nicoletti, 2005). Sin embargo, diez años después se situaba cerca de los menos restrictivos (Wölfl et al., 2009), obteniendo resultados mejores que la media en los antiguos indicadores sobre control estatal, barreras al emprendimiento y barreras al comercio y la inversión. Así, en estos años la puntuación en el indicador general pasó de 2,39 en 1998 a 1,44 en 2013 (Gráfico 1) ${ }^{5}$, lo que refleja los avances en este periodo en términos de introducción progresiva de medidas procompetitivas y estándares de buena regulación (las cifras más bajas reflejan una intensidad regulatoria menor).

En 2018, y tras el cambio estructural del indicador general, España ha obtenido una puntuación de 1,07, situándose en el ranking de países de la OCDE en segunda posición junto a Dinamarca, solo por detrás de Reino Unido (Gráfico 2). En concreto, ha obtenido una puntuación de 1,30 en el indicador de nivel alto sobre distorsiones introducidas por el Estado y una puntuación de 0,84 en el indicador $\triangleright$

5 Por simplicidad expositiva se ha incluido la puntuación de 2018, pero debemos recordar que, debido a los cambios metodológicos, no es directamente comparable con la de años anteriores. 
sobre barreras de entrada. Si profundizamos en cada uno de estos indicadores de alto nivel, podemos observar grandes diferencias entre los distintos indicadores de nivel medio (Gráfico 2).

Por ejemplo, si nos fijamos en los indicadores que componen el subindicador de alto nivel de distorsiones introducidas por el Estado, España se sitúa en tercera $(1,44)$ y quinta posición $(0,93)$ en los indicadores sobre propiedad pública y simplificación y evaluación de la regulación, respectivamente; ocupando el puesto vigésimo sexto (1,53 puntos) en el indicador sobre participación del Estado en operaciones comerciales.

Las barreras de entrada son más altas en el ámbito de los sectores de red y de servicios $(1,92)$ que en el área comercial y de inversiones $(0,45)$, obteniendo especialmente buenos resultados en el ámbito de creación de empresas gracias a las novedades introducidas por la Ley 14/2013, de 27 de septiembre, de apoyo a los emprendedores y su internacionalización, donde España se sitúa en cuarta posición con una puntuación de solo 0,19 puntos.

\section{Indicadores sectoriales. Comparación 2013-2018}

Leyes como la 39/2015, de 1 de octubre, del Procedimiento Administrativo Común de las Administraciones Públicas, o la 40/2015, de 1 de octubre, de Régimen Jurídico del Sector Público, han introducido preceptos para impulsar la buena regulación, mejorando la posición de España en los indicadores sectoriales. A esto se añade la reducción de barreras (tanto para la creación de startups como al comercio e inversión) incentivada por la Ley 14/2013, de 27 de septiembre, y otras modificaciones en leyes sectoriales.
Debido a las novedades metodológicas, no es posible realizar una comparación del indicador general. No obstante, se pueden avanzar comparaciones más fiables para algunos indicadores sectoriales donde la composición del indicador no ha cambiado.

Las últimas reformas de la regulación tanto del sector de la electricidad ${ }^{6}$ como del sector gasista ${ }^{7}$ están orientadas a fomentar la transparencia en los mercados y a introducir competencia en los mercados, permitiendo la entrada de nuevos operadores y mayor libertad de elección para los consumidores.

En telecomunicaciones España se encuentra entre los países con una mejor puntuación, 0,24 puntos, ocupando en el ranking la séptima posición. En este indicador se observa un importante avance impulsado por las novedades ${ }^{8}$ introducidas por la Ley 9/2014, de 9 de mayo, General de Telecomunicaciones, y el Real Decreto 123/2017, de 24 de febrero, por el que se aprueba el Reglamento sobre el Uso del Dominio Público Radioeléctrico, que permite crear un mercado secundario para el espectro radioeléctrico, lo que fomenta la incorporación de nuevos entrantes. Adicionalmente, existen $\triangleright$

\footnotetext{
6 La Ley 24/2013, de 26 de diciembre, del Sector Eléctrico reconoce la libre iniciativa empresarial para el ejercicio de las actividades de generación, comercio, transporte, distribución y suministro al por menor de electricidad. Esta ley también regula el acceso de las empresas a la red fomentando la competencia y la posibilidad de que los consumidores elijan a su proveedor, lo que incide positivamente en la estructura del mercado y reduce barreras a la competencia, así como prevé mecanismos de adaptación a la demanda por parte de los grandes usuarios industriales o comerciales.

Adicionalmente, la Resolución de 23 de mayo de 2014 de la Dirección General de Política Energética y Minas establece el contenido mínimo y el modelo de factura eléctrica que deben ser remitidas a los consumidores, incluyendo información clara sobre su consumo y las tarifas aplicadas. Solamente el «precio voluntario al pequeño consumidor» está regulado por el Gobierno, pero existe una tarifa para consumidores vulnerables (bonus social) que introduce ganancias de bienestar para el consumidor.

7 En el sector gasista el Real Decreto 984/2015, de 30 de octubre, por el que se regula el mercado organizado de gas y el acceso de terceros a las instalaciones del sistema de gas natural, favorece las actividades de almacenamiento, distribución y transmisión de gas, reduciendo el poder de mercado en el sector.

8 Entre ellas destaca la conservación del número de teléfono fijo o móvil por los abonados con independencia del operador que preste el servicio, favoreciendo así al consumidor.
} 


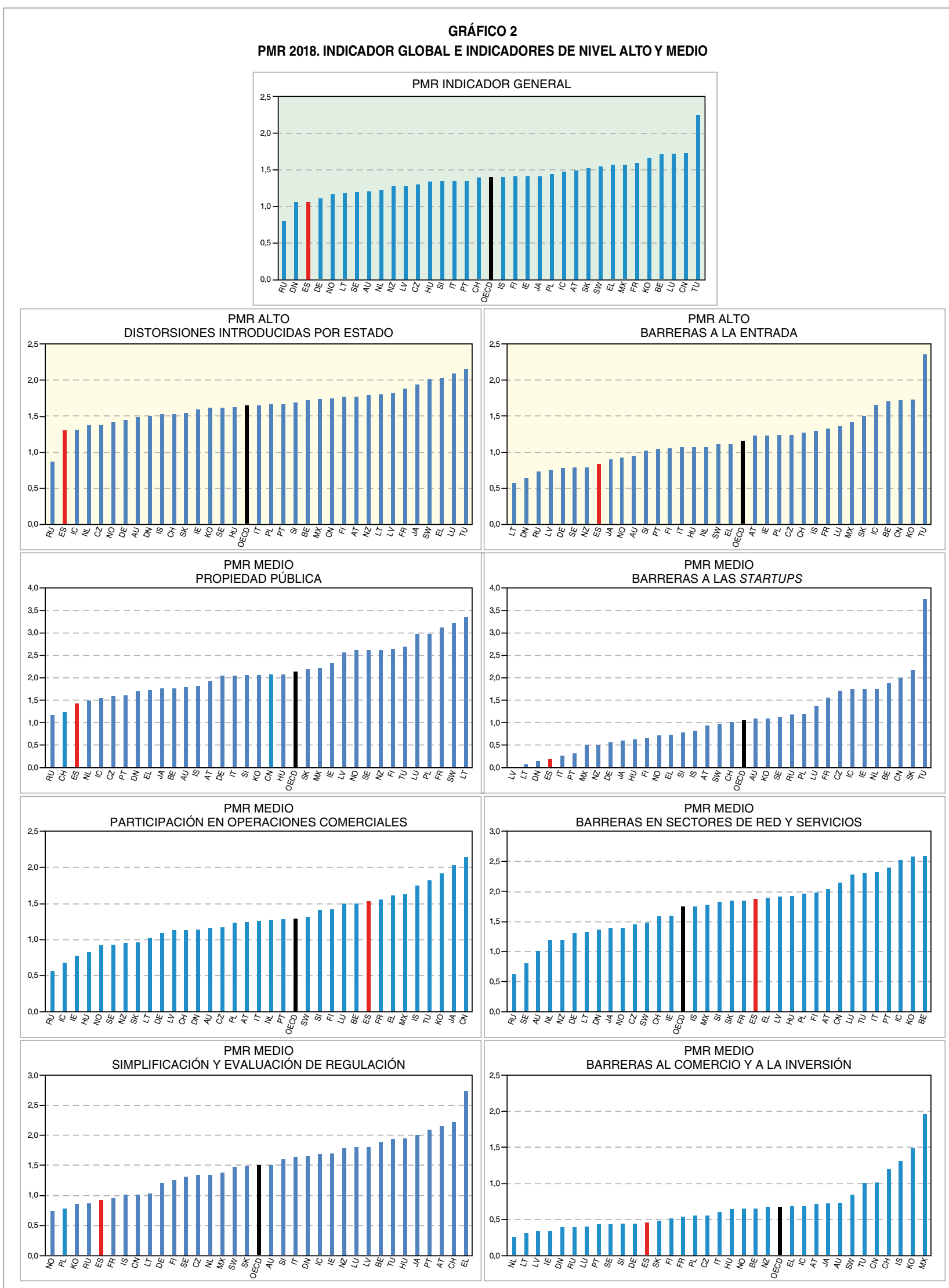

Fuente: elaboración propia a partir de OCDE, 2019. 
diversas resoluciones de la CNMC que imponen obligaciones específicas a los operadores con poder significativo en los mercados mayoristas y minoristas de redes fijas y móviles.

En cuanto al sector de transportes, se observa una tendencia generalizada en todos los países a mantener regulaciones más restrictivas. España se sitúa mejor que la media de la OCDE, con una puntuación de 1,69, siendo llamativa la disparidad de resultados por tipo de transporte. En transporte marítimo ocupa la primera posición, con 0,38 puntos, y en transporte aéreo, con una puntuación menor $(0,35)$, ocupa la quinta posición. En contraposición, en transporte ferroviario España se encuentra entre los cinco países con una regulación más restrictiva y en transporte por carretera, a pesar de situarse mejor que la media, se posiciona en el decimotercer puesto, con una puntuación de 1,35.

En distribución comercial España se sitúa entre los diez países con una regulación más restrictiva (puntuación de 1,69), aunque ha mejorado considerablemente respecto a 2013 por la introducción de principios e instrumentos de buena regulación ${ }^{9}$, así como por el principio de no exigencia de autorizaciones adicionales para la apertura de establecimientos ${ }^{10}$. Sin embargo, obtiene una de las puntuaciones más altas, con 4,75 puntos, en el área de venta y distribución al por menor de medicamentos.

Destaca el impacto positivo que ha tenido, en la puntuación de España, la introducción de profesiones como contables y agentes de la propiedad inmobiliaria, en las que se ha obtenido una puntuación cercana a cero, frente a la elevada puntuación, 4,96, obtenida en la profesión de notarios (también introducidos en 2018).

9 Leyes 39 y 40/2015, de 1 de octubre.

10 Debido a la trasposición de la Directiva de Servicios en el Real Decreto Ley $8 / 2014$, sobre medidas urgentes para el crecimiento, la competitividad y la eficiencia.
En ingenieros civiles, arquitectos y abogados, se obtiene una puntuación siempre por debajo de la media de la OCDE, de 0,95, 1 y 1,52, respectivamente. Es destacable la mejora de la puntuación en ingenieros y arquitectos debido a la nueva normativa para la acreditación de cualificaciones profesionales ${ }^{11}$.

Por tanto, a pesar de que en conjunto España tenga un buen resultado en el indicador general, es importante prestar atención a ciertos sectores como el de transportes (en concreto, transporte ferroviario y por carreteras), distribución comercial o servicios profesionales, cuya regulación influye negativamente no solo en los indicadores sectoriales, sino en el funcionamiento general de los mercados de bienes y servicios.

En el Gráfico 3 se incluyen los indicadores en los que se ha producido una mejora.

\section{Retos pendientes}

El principal objetivo de los indicadores PMR es ayudar a los países a identificar aquellas áreas en las que la regulación no favorece comportamientos procompetitivos. Para España, los resultados del PMR 2018 permiten identificar, probablemente sin sorpresas para el lector, cuatro áreas o sectores en los que la introducción de reformas puede dinamizar la competencia: sector ferroviario, transporte por carretera, venta y distribución al por menor de medicamentos, y servicios profesionales prestados por notarios. Precisamente, las tres últimas áreas han sido introducidas o reforzadas en 2018, D

\footnotetext{
11 Real Decreto 967/2014, de 21 de noviembre, por el que se establecen los requisitos y el procedimiento para la homologación y declaración de equivalencia a titulación y a nivel académico universitario oficial y para la convalidación de estudios extranjeros de educación superior, y el procedimiento para determinar la correspondencia a los niveles del marco español de cualificaciones para la educación superior de los títulos oficiales de Arquitecto, Ingeniero, Licenciado, Arquitecto Técnico, Ingeniero Técnico y Diplomado.
} 


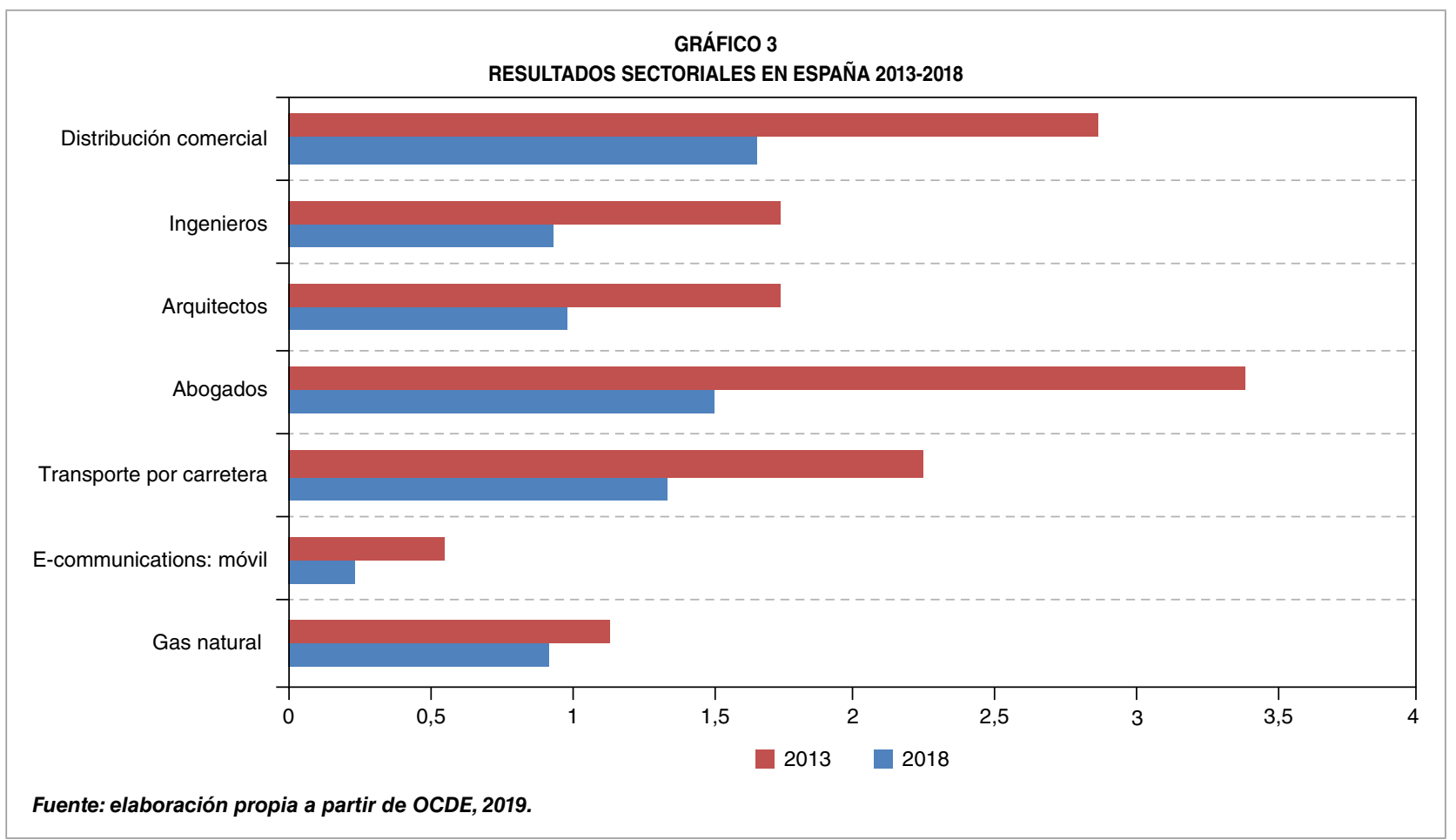

lo cual refleja cómo las reformas metodológicas buscan llegar a áreas del mercado en las que todavía hay margen para la mejora.

\subsection{Transporte ferroviario}

El sector ferroviario, actualmente de propiedad pública, es controlado y fiscalizado por el Estado, de modo que incluso la venta de acciones debe ser aprobada por ley. No obstante, lo más destacable, según la OCDE, es la falta de competencia en la provisión de servicios de transporte de viajeros y en el sistema de presentación de ofertas. Áreas en las cuales ha habido novedades importantes posteriores a 2018 y, por tanto, no capturadas en el PMR.

En este contexto es fundamental fomentar la introducción de mecanismos procompetitivos. La CNMC había recomendado «mantener la separación estructural de ADIF y ADIF Alta Velocidad respecto de RENFE asegurando la plena autonomía de cada una de ellas» (CNMC,
2019). Adicionalmente, el informe señala que, dada la existencia de estructuras verticales integradas, se debería contar con una gestora independiente que garantice la igualdad en el acceso a las infraestructuras ferroviarias a todos los operadores de la red para prevenir la discriminación. Por ejemplo, como señala la CNMC, «la entrada de nuevos operadores está condicionada a la obtención de capacidad en la infraestructura ferroviaria, por lo que se requiere una correcta gestión de la infraestructura que maximice la capacidad disponible y la adjudique a los operadores conforme a un procedimiento no discriminatorio establecido previamente».

La necesaria liberalización del transporte de viajeros ya se ha advertido a nivel europeo. El cuarto paquete ferroviario traspuesto en $2018^{12}$ al ordenamiento jurídico interno ha impulsado la apertura del mercado de los servicios $\triangleright$

12 Real Decreto Ley 23/2018, de 21 de diciembre, de transposición de directivas en materia de marcas, transporte ferroviario y viajes combinados y servicios de viaje vinculados que modifica la vigente Ley $38 / 2015$, de 29 de septiembre, del sector ferroviario. 


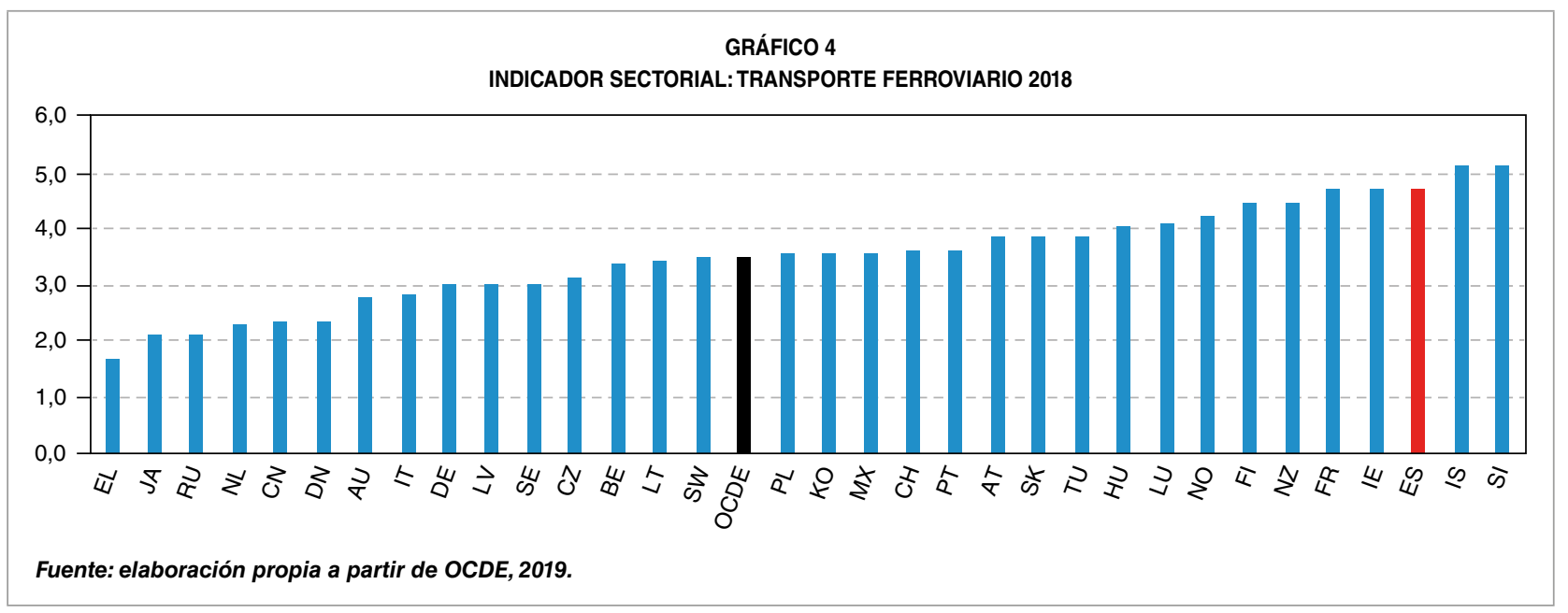

nacionales de transporte de viajeros por ferrocarril y la imparcialidad en la gobernanza de las infraestructuras ferroviarias, y ha reforzado las competencias de la CNMC como órgano supervisor de la competencia en los mercados de servicios ferroviarios, en concreto en el transporte de viaje en alta velocidad. El año 2020 es la fecha de inicio establecida para la entrada de nuevas empresas ferroviarias en el transporte de viajeros por ferrocarril en los servicios de larga distancia y alta velocidad.

A partir de dicha fecha, cualquier empresa que disponga de la licencia de empresa ferroviaria y del certificado de seguridad, que otorga la Agencia Estatal de Seguridad Ferroviaria, y haya solicitado el uso de la infraestructura ferroviaria al administrador de infraestructuras podrá prestar servicios en competencia con Renfe.

\subsection{Transporte por carretera}

En este sector son diversos los aspectos que hacen que España tenga peores resultados que la media de la OCDE.

En primer lugar, en lo referido a barreras de entrada, el PMR, en línea con la corriente de la better regulation, valora negativamente la exigencia de autorización para la constitución tanto de empresas de transporte de mercancías como de transporte nacional de viajeros de larga distancia. Sin embargo, en términos comparativos, todos los países participantes exigen autorización en ambos casos, salvo Chile (que exige notificación) y Australia (que solo permite la notificación para el transporte de mercancías).

Sin embargo, lo que verdaderamente afecta de manera negativa a España es que, a diferencia de la gran mayoría de países de la OCDE, el número de compañías que pueden prestar servicios de transporte nacional de viajeros de larga distancia se encuentra restringido. Esto se debe a que la prestación de este tipo de servicios de carácter público se articula a través de una concesión por medio de un contrato de gestión ${ }^{13}$, lo que conlleva a su vez la regulación de las tarifas y la previa aprobación de todas las nuevas rutas que una empresa quiera cubrir ${ }^{14}$. Este modelo de explotación ya ha sido $\triangleright$

13 El artículo 71 de la Ley 16/1987, de 30 de julio, de Ordenación de los Transportes Terrestres (LOTT) en su párrafo segundo establece: «Como regla general, la prestación de los mencionados servicios [de transporte público regular de viajeros de uso general] se llevará a cabo por la empresa a la que la Administración adjudique el correspondiente contrato de gestión...".

14 Artículo 70 de la LOTT. 


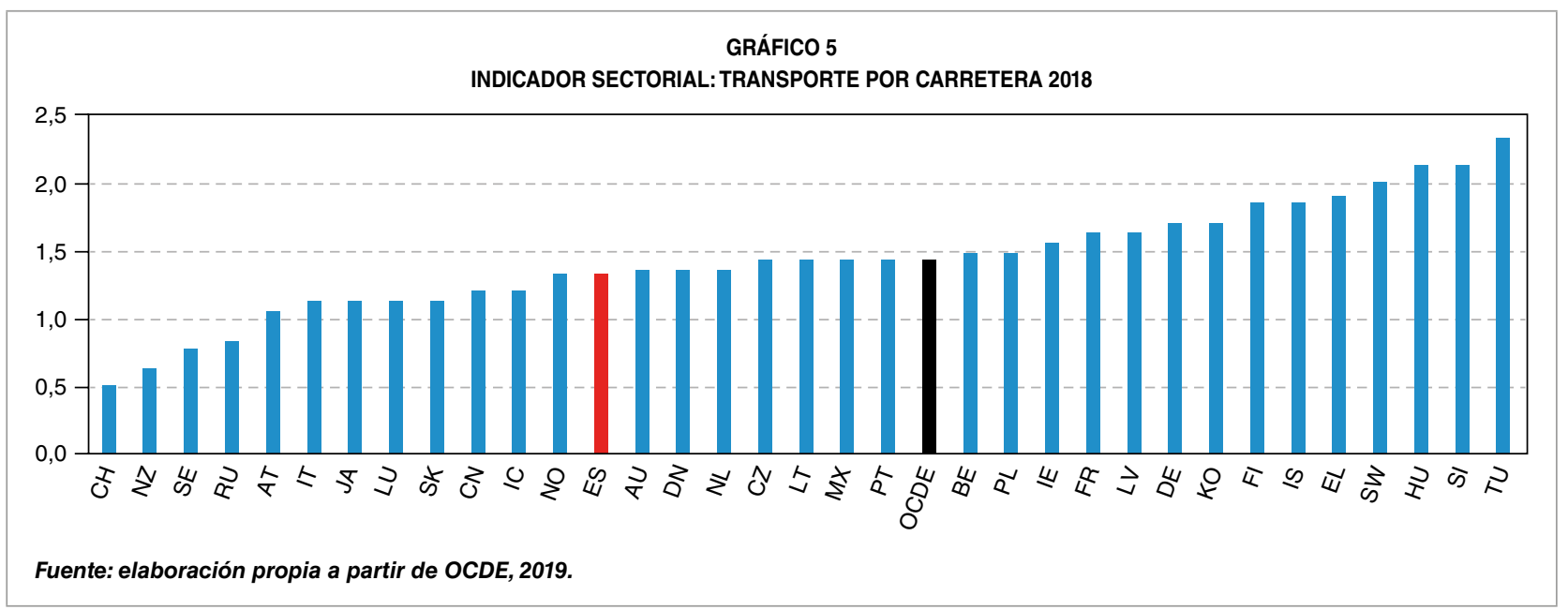

cuestionado en varias ocasiones por la CNMC y su predecesora, la CNC, que considera que el uso de la concesión supone negar la competencia en la prestación del servicio, dado que, en aquellos casos en los que exista suficiente demanda sería eficiente que varios operadores pudieran desarrollar el servicio en condiciones de rentabilidad, permitiendo el libre establecimiento de precios en el mercado (CNC, 2012).

Por otra parte, en relación con el transporte por carretera, España se ve penalizada en el indicador general por la diferente regulación aplicable a taxis y VTC ${ }^{15}$. En esta área, el PMR no penaliza tanto las restricciones a la prestación del servicio como la distinta regulación y requisitos exigidos tanto en el acceso como en el ejercicio de la actividad a taxis y VTC. Sin pretender analizar en detalle la regulación de estos servicios, pues es abundante y excede el objeto de este artículo ${ }^{16}$, podemos destacar las diferencias también señaladas por la CNMC (CNMC, 2018) en cuanto a las tarifas («los taxis deben aplicar

15 Utilizaremos la denominación VTC para referirnos a arrendamientos de vehículo con conductor.

16 Es una materia en la que coexiste normativa estatal, autonómica y local y en la que, además, el reparto competencial varía en función del servicio de que se esté hablando. Los PMR 2018 no recogen las reformas de la LOTT introducidas por los Reales Decretos Leyes 3/2018, de 20 de abril, y 13/2018, de 28 de septiembre, en materia de arrendamiento de vehículos con conductor. tarifas fijadas administrativamente, ya sea tarificación fija o de máximos, mientras que los VTC se someten a precios libres»), las formas de contratación del servicio («mientras los taxis pueden contratarse en paradas, directamente en la calle, mediante la precontratación telefónica u otra tecnología de comunicación, los VTC solamente puedan prestar servicio mediante la precontratación» ${ }^{17}$ ), el uso de los carriles bus (determinadas disposiciones locales facilitan el ejercicio de la actividad del taxi mediante el acceso a carriles [carriles bus] o zonas de circulación restringida [...] que, o bien no están disponibles para el VTC, o se admiten con distinta intensidad „ ${ }^{18}$ ), o los requisitos de acceso a la profesión («en términos generales, a los conductores de VTC no se les exige ningún tipo de requisito, más allá de la licencia de conducción, mientras que a los taxistas, dependiendo del lugar en el que presten sus servicios, se les exige superar un examen de aptitud, tener el título de educación secundaria obligatoria o tener unos determinados conocimientos lingüísticos, entre otros»).

17 Artículo 182.1 del Real Decreto 1211/1990, de 28 de septiembre, por el que se aprueba el Reglamento de la Ley de Ordenación de los Transportes Terrestres.

18 Informe de 17 de enero de 2018 sobre barreras al ejercicio de la actividad de alquiler de vehículo con conductor debido a restricciones impuestas en ciertas localidades (UM/145/17). 


\subsection{Distribución y venta al por menor de medicamentos}

Los resultados del PMR sitúan a España entre los tres países con una regulación más restrictiva en cuanto a la distribución y venta de medicamentos al por menor. Por una parte, el PMR destaca las restricciones relacionadas con la apertura de establecimientos farmacéuticos y, por otra, las restricciones relativas a la venta de medicamentos.

En lo que se refiere a las primeras, existen limitaciones en cuanto al número de farmacias que pueden abrirse en un área específica, el lugar en el que deben situarse, quién puede ser propietario (únicamente licenciados en Farmacia) y cuántas farmacias se pueden poseer $^{19}$ (solamente una) ${ }^{20}$. Por tanto, las oficinas de farmacia compiten fundamentalmente en variables distintas del precio, que les viene dado, como la calidad del servicio (entrega a domicilio, tiempo de desplazamiento hasta el establecimiento, velocidad de dispensación, sistemas personificados de dosificación, etcétera) o los horarios de apertura ${ }^{21}$.

En 2015, un estudio de la CNMC concluyó que las restricciones de acceso y ejercicio de la actividad son ineficientes y perjudican a la competencia y al interés general (CNMC, 2015). Desde esta perspectiva se realizaron ciertas recomendaciones en aspectos similares a los que señala el PMR, como la supresión de las restricciones sobre módulos de población y

\footnotetext{
19 Esta estricta regulación partiría de la Ley 16/1997, de 25 de abril, de Regulación de Servicios de las Oficinas de Farmacia, que en su primer artículo sostiene que las oficinas de farmacia tienen la consideración de establecimientos sanitarios privados, de interés público y, por supuesto, de la salud pública como bien público de necesaria protección.

20 Artículo 103 de la Ley 14/1986, de 25 de abril, General de Sanidad.

21 El artículo 6 de la Ley 16/1997, de 25 de abril, establece que las oficinas de farmacia prestarán sus servicios en régimen de libertad y flexibilidad, sin perjuicio del cumplimiento de los horarios oficiales y normas sobre guardias, vacaciones, urgencias y demás circunstancias derivadas de la naturaleza de su servicio, fijadas por las comunidades autónomas, al objeto de garantizar la continuidad de la asistencia.
}

las distancias mínimas obligatorias, tanto entre oficinas de farmacia como entre estas y los centros de salud. También propuso eliminar el requisito de que los propietarios y titulares de oficina de farmacia sean exclusivamente titulados en Farmacia, de forma que exista libertad en la propiedad de las farmacias así como en la propiedad de más de una.

Por otra parte, el PMR valora negativamente las restricciones a la venta de medicamentos. En España, tanto los medicamentos sujetos a prescripción como los no sujetos a prescripción pueden venderse únicamente en farmacias. De estos, solo los no sujetos a prescripción pueden venderse online, a pesar de que en el ámbito europeo está permitida la venta online de medicamentos sujetos a prescripción ${ }^{22}$. Además, el PMR destaca la prohibición de anunciar los descuentos en precio que pueden realizarse en algunos medicamentos no sujetos a prescripción (si bien se pueden aplicar los descuentos, las farmacias no pueden publicitarlos como reclamo para la venta) ${ }^{23}$. En el informe de 2015, la CNMC recomienda para los medicamentos no sujetos a prescripción médica eliminar la reserva de actividad de las oficinas de farmacia en la dispensación.

\subsection{Notarios}

En el sector de los servicios profesionales, en términos generales, España se ve perjudicada por el gran número de reservas de $\triangleright$

\footnotetext{
22 Directiva 2000/31/CE del Parlamento Europeo y del Consejo, de 8 de junio de 2000, relativa a determinados aspectos jurídicos de los servicios de la sociedad de la información, en particular el comercio electrónico en el mercado interior (Directiva sobre el comercio electrónico).

23 Véase aclaración en el documento de la Agencia Española de Medicamentos y Productos Sanitarios (AEMPS) titulado «Preguntas y respuestas para las farmacias sobre venta a distancia de medicamentos de uso humano no sujetos a prescripción médica». Disponible en: https:// distafarma.aemps.es/farmacom/faces/templates/estaticas/informacion. xhtm/\#
} 


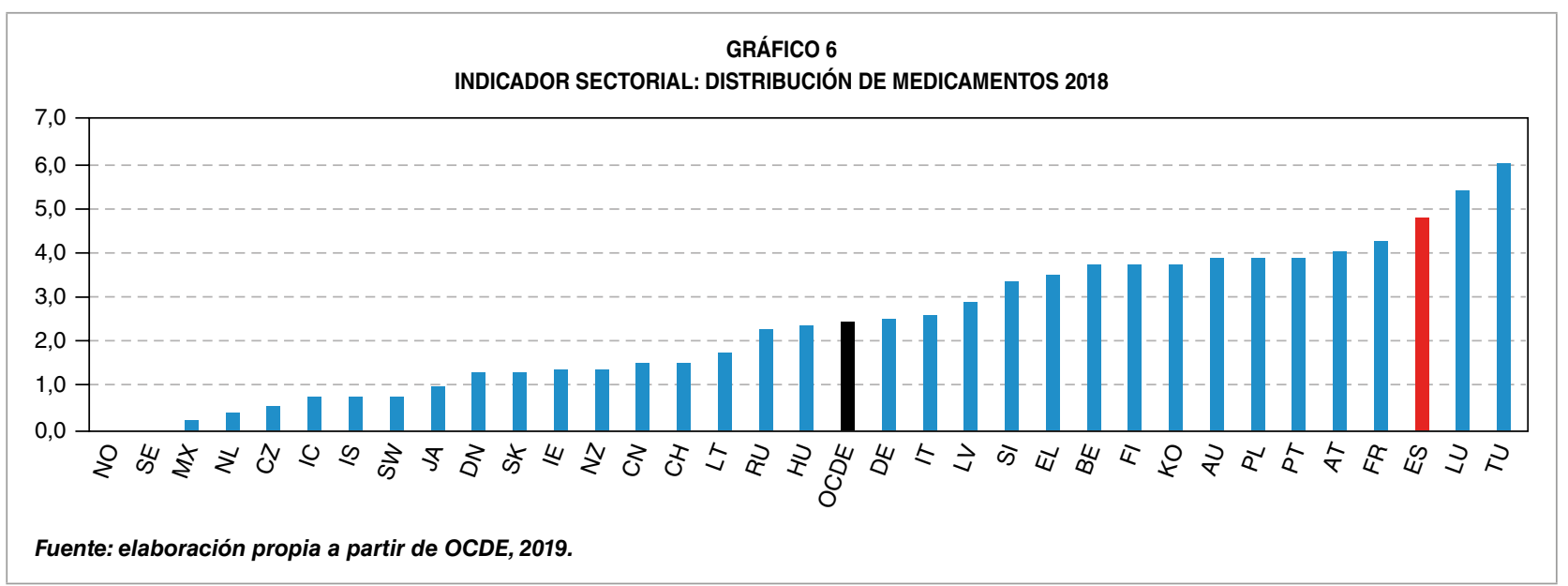

actividad atribuidas a ingenieros, abogados y arquitectos, a lo que se añade la exigencia de colegiación obligatoria. En este sentido, ya existe un mandato para el Gobierno de aprobación de un proyecto de ley que determine las profesiones para cuyo ejercicio es obligatoria la colegiación, cuya puesta en marcha está todavía pendiente ${ }^{24}$.

En lo que respecta a los notarios, la puntuación de España es alta $(4,96)$, como en la mayoría de países en los que existe esta profesión, variando de 3,54 en Francia a 5,43 en Alemania. De hecho, en casi todas las preguntas sobre esta profesión España obtiene la máxima puntuación, es decir, se trata de una profesión muy regulada. Esto se debe a que los notarios, de acuerdo con el artículo 1 del Reglamento de la Organización y Régimen del Notariado25, son a la vez funcionarios públicos y profesionales del derecho, lo cual sustrae de la competencia bastantes aspectos de su régimen ${ }^{26}$. Esta

\footnotetext{
24 Disposición transitoria cuarta de la Ley 25/2009, de 22 de diciembre, de modificación de diversas leyes para su adaptación a la Ley sobre el Libre Acceso a las Actividades de Servicios y su Ejercicio.

25 Aprobado por Decreto de 2 de junio de 1944

26 El artículo 4 del Real Decreto Legislativo 5/2015, de 30 de octubre, por el que se aprueba el texto refundido de la Ley del Estatuto Básico del Empleado Público, los menciona dentro de la categoría de personal con legislación específica propia, y el artículo 1 de la Ley del Notariado de 28 de mayo de 1862 los define como funcionarios públicos autorizados para dar fe, conforme a las leyes, de los contratos y demás actos extrajudiciales.
}

situación es la que hace que el número de titulados que pueden ejercer la profesión se encuentre limitado por ley, que existan restricciones territoriales al ejercicio o que los precios de sus servicios así como la publicidad de los mismos se encuentren regulados. También conlleva restricciones en cuanto a la forma legal que puede adoptar una notaría, la prohibición de ejercicio en asociación con otros profesionales, así como en cuanto a quién puede ser propietario de una notaría o tener derechos de voto en ella.

Sin embargo, si se observa el Gráfico 7, se puede apreciar que esta profesión no existe en todos los países. Tal y como señala la OCDE, es concretamente en países de derecho continental donde los notarios existen y realizan tareas administrativas y judiciales por delegación del Gobierno. Los resultados de los PMR corroboran la existencia de dos modelos: continental y anglosajón, siendo, por el propio diseño del índice, mejor valorado este último. A juicio de las autoras, el objetivo de estos indicadores es incentivar a los países a implementar mejoras en la regulación, pero debido a la dualidad de sistemas, de la comparación de las regulaciones en estos servicios, es difícil extraer mejores prácticas. 


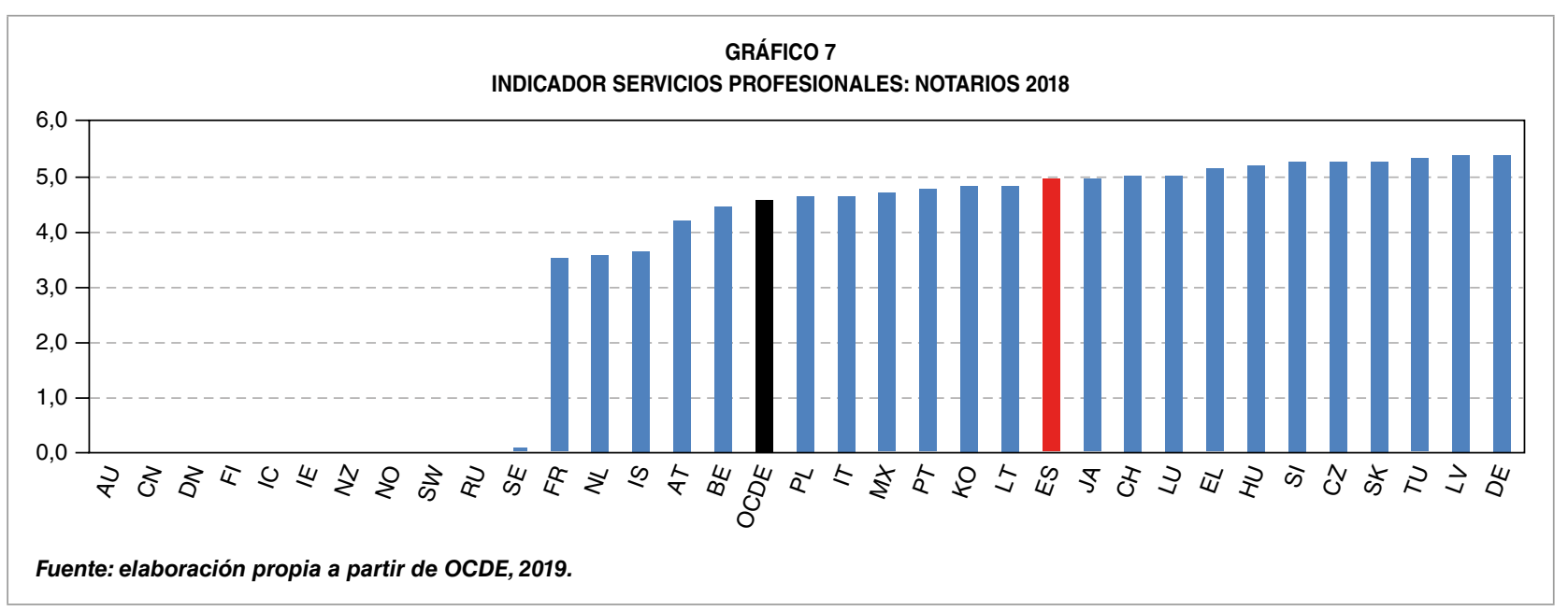

\section{Conclusión}

Los resultados de los PMR en 2018 reflejan una mejora sustancial de España, causada tanto por las reformas implementadas en los últimos años como por los cambios metodológicos y de contenido introducidos en esta última edición. Con todo, existen sectores en los que desde el punto de vista de la competencia queda mucho camino por recorrer. En algunos de ellos se requiere de mayor impulso reformista dado que el cuestionamiento del sistema por parte de la sociedad es claro. En otros, el consenso sobre las líneas de avance está aún lejano.

\section{Agradecimientos}

Las autoras agradecen especialmente a Luis González Calbet su ayuda y asesoramiento, así como las sugerencias y comentarios de Luis Martí Álvarez y Noa Laguna Goya.

\section{Bibliografía}

CNC (2012). Anteproyecto de ley por el que se modifica la Ley de ordenación de los transportes terrestres. IPN 75/12. Recuperado de https:// www.cnmc.es/sites/default/files/424386_6.pdf

CNMC (2015). Estudio sobre el mercado de distribución minorista de medicamentos en España. E/CNMC/003/15. Recuperado de https://www. cnmc.es/expedientes/ecnmc00315

CNMC (2017). PRD por el que se modifican diversas normas reglamentarias para adaptarlas a la Ley de Ordenación de los Transportes Terrestres y a los cambios introducidos en la reglamentación de la UE. IPN/CNMC/018/17. Recuperado de https://www.cnmc.es/sites/default/files/18789 26_31.pdf

CNMC (2018a). Sobre el Real Decreto Ley 13/2018, de 28 de septiembre, que modifica la LOTT en materia de arrendamiento de vehículos con conductor. PRO/CNMC/003/18. Recuperado de https://www.cnmc.es/expedientes/ procnmc00318

CNMC (2018b). Informe de 17 de enero de 2018 sobre barreras al ejercicio de la actividad de alquiler de vehículo con conductor debido a restricciones impuestas en ciertas localidades. UM/ 145/17. Recuperado de https://www.cnmc.es/sites/default/files/1902849_12.pdf

CNMC (2019). Estudio sobre la liberalización del transporte de viajeros por ferrocarril. E/CNMC/ 004/19. Recuperado de https://www.cnmc.es/expedientes/ecnmc00419-0 
Conway, P., Janod, V., y Nicoletti, G. (2005). Paper Product market regulation in OECD Countries: 1998 to 2003. OECD Economics Department Working Papers (419). París: OECD Publishing.

Conway, P., De Rosa, D., Nicoletti, G., y Steiner, F. (2006). Regulation, competition and productivity convergence. OECD Economics Department Working Papers (509). París: OECD Publishing.

Gunningham, N., Grabosky, P., y Sinclair, D. (1998). Smart Regulation: Designing Environmental Policy. Oxford: Oxford University Press.

Leibenstein, H. (1966). Allocative Efficiency vs. «X-Efficiency». The American Economic Review, 56(3), 392-415.

Mandelkern Group on Better Regulation (2001). Informe Mandelkern.

Meyer, M., y Vickers, J. (1997). Performance Comparisons and Dynamic Incentives. Journal of Political Economy, 105(3), 547-581.

Nicoletti, G., Scarpetta, S., y Boylaud, O. (2000). Summary indicators of product market regulation with an extension to employment protection legislation. OECD Economics Department Working Papers (226). París: OECD Publishing.

Nicoletti, G., y Scarpetta, S. (2003). Regulation, productivity and growth: OECD Evidence. OECD Economics Department Working Papers (347). París: OECD Publishing.

OECD (2018). Preliminary results for the 2018 Product market regulation indicators. ECO/CPE/ WP1(2018)19.

OCDE (2019). Resultados de los indicadores PMR. Recuperado de https://www.oecd.org/economy/ reform/indicators-of-product-market-regulation/

Winston, C. (1993). Economic deregulation: days of reckoning for microeconomists. Journal of Economic Literature (31), 1263-1289.

Wölfl, A., Wanner, I., Kozluk, T., y Nicoletti, G. (2009). 10 years of product market reform in OECD-Countries: Insight from a revised PMR indicator. OECD Economics Department Working Papers (695). París: OECD Publishing.

ANEXO

Relación de siglas

\begin{tabular}{|llll|}
\hline Australia & AU & Corea del Sur & KO \\
Austria & AT & Letonia & LV \\
Bélgica & BE & Lituania & LT \\
Canadá & CN & Luxemburgo & LU \\
Chile & CH & México & MX \\
Chequia & CZ & Países Bajos & NL \\
Dinamarca & DN & Nueva Zelanda & NZ \\
Finlandia & FI & Noruega & NO \\
Francia & FR & Polonia & PL \\
Alemania & DE & Portugal & PT \\
Grecia & EL & República Eslovaca & SK \\
Hungría & HU & Eslovenia & SI \\
Islandia & IC & España & ES \\
Irlanda & IE & Suecia & SE \\
Israel & IS & Suiza & SW \\
Italia & IT & Turquía & TU \\
Japón & JA & Reino Unido & RU \\
\hline
\end{tabular}

